\title{
Underlying causes of cerebral palsy: public health perspectives
}

\author{
Gulnara Kapanova ${ }^{1}$, Shynar Malik ${ }^{1}$, Aima Adylova ${ }^{2}$ \\ ${ }^{1}$ Al Farabi Kazakh National University, Kazakhstan, ${ }^{2}$ Kazakhstan School of Public Health, Kazakhstan
}

\begin{abstract}
Cerebral palsy (CP) is a neurological pathology that is characterized by a combination of signs and symptoms that occur in neurodegenerative or metabolic disorder during the first few years of life. It is a complex pathology orchestrated by a plethora of different causes. The current diagnostic regimen for CP involves brain magnetic resonance imaging (MRI), and antenatal and perinatal insult. Despite advances in the field of genetics and molecular biology, the evaluating the underlying causes of this severe pathology are still bleak. In this review we have attempted to provide a landscape of the underlying mechanisms of cerebral palsy. We have partitioned this review broadly into genetic and proteomic-based studies, which have enriched our understanding about the pathogenesis of CP.
\end{abstract}

Key words: cerebral palsy, neuropathology, disease.

\section{Introduction}

Cerebral palsy (CP) is a major neurodevelopmental disorder that has been reported to affect 1 in 500 children. The causative factors and aetiology of this particular pathology are still obscure [3]. Despite its complex aetiology, magnetic resonance imaging (MRI) has remained an efficient way to diagnose CP. In addition to this hypoxia-ischaemia, placental insufficiency and prenatal infections are well-reported causes to diagnose CP. Despite these advents, the exact cause of this complex disease is still illusive $[9,35]$. Hypoxic ischaemic injury has been affiliated with the development of CP; it has been estimated that intrapartum hypoxia ischaemia is responsible for nearly $10 \%$ cases of CP [43]. Although there have been improvements in obstetric practices, and better antenatal and perinatal care, the incidence rate of CP has increased over the past decades. This has led to the speculation that there are unknown patho- physiological processes that are responsible for the significant elevation of CP cases worldwide. New research has shed light on the involvement of genetic and epigenetic factors in the development of CP. These factors have been estimated to play a significant role in disease aetiology and prevalence. Nearly $30 \%$ cases of CP have been reported to involve genetic changes [20]. There has been a growing consensus that dictates the involvement of genetic contribution in the development of CP. Characteristically 4 types of genetic variations play a vital role in the pathogenesis of CP [20]. Understanding these variations can open up new avenues in the detection diagnosis and treatment of CP. Genetic mutations are a continuous threat to the normal cellular function and can trigger aberrant expression of proteins, which ultimately leads to loss of normal cellular functionality. In addition to this genetic predisposition are the main events that determine the genetics 
of CP. Despite their clear involvement in the development of CP, very few studies have been published that address the genetic basis of CP. Advancements in the field of molecular biology, genome-wide association studies (GWAS), RNA-seq, proteomics, and RNAi have brought us a step closer to unravelling the complexities of the human genome and pinpointing genetic variations responsible for the development of various genetic diseases [33]. Despite this understanding, the molecular basis of $\mathrm{CP}$ remains unknown. Aberrant neurobiological pathways are the hallmark of CP [20]. Therefore, understanding the genetic aberrations responsible for triggering these neurobiological pathways seems a promising diagnostic/prognostic approach for CP. CP is a complex disease that involves a number of genetic variations and deregulated protein networks, which trigger oxidative stress, inflammation, and ischaemia, leading to neuronal death [8]. Similarly to other neuronal motor diseases such as autism and intellectual disability, understanding the genetics can aid in the development of a framework for the elaboration of the fundamental neurobiological pathways involved in CP [10]. Interestingly, findings to date indicate that a plethora of genes are involved in the development of CP and the majority of these genes are still undiscovered [10]. Genetic mutations in combination with environmental factors determine the outcome of a mutation. Some genetic variations, such as deleterious mutations, are less significant because they do not disrupt protein function and have less profound effects on genes while some mutations are lethal because they profoundly distort the protein and lead to major effects. However, it has been reported that deleterious mutations in combination with environmental factors can transform less damaging mutations into more adverse anomalies. Therefore, some infants with severe variations survive for a relatively long time, while infants having minor variations may not survive for long. This has been well observed in animal models developed for CP.

Epigenetic modifications play a significant role in wide-ranging diseases [12].

Certain exciting studies have provided clues about important genes that may epigenetically contribute to the development and occurrence of cerebral palsy in monozygotic twins [19]. In another study, a team of researchers identified differentially methylated regions in monozygotic twins discordant for CP [31]. However, clinicians still have an incomplete under- standing about detailed epigenetic landscape that operates during pathology of cerebral palsy.

Oxidative stress and hypoxia play critical roles in different diseases [29,37]. Likewise, oxidative stress is a causative factor in cerebral palsy. $\mathrm{N}$-acetylcysteine (NAC) was conjugated to PAMAM dendrimers (D-NAC) for the drug delivery across the blood-brain barrier into cells linked with neuro-inflammation [48]. Neuroinflammatory models with newborn motor deficits that mimicked human CP were generated by injection of Escherichia coli endotoxin into the uterus of pregnant rabbit dams. Accordingly, within 6 hours of birth, D-NAC was administered intravenously into the kits. Importantly, the kits that received D-NAC demonstrated significantly improved motor control, hypertonia, and coordination [48].

Noteworthy breakthroughs in functional genomics and proteomics have dramatically enhanced our concepts about contributory role of cell signalling pathways in development and diseases [11,13,14,16,17].

Accumulating evidence has shed light on the fact that alteration in the typical molecular signalling pathways specifically involved in the transcription, translation, synaptic function, development of cellular infrastructure, circuitry, neuronal glial signalling, and inflammation can lead to human disease, and their outcomes can be studied in animal models [4]. This can be accurate for CP as well, and devising a genetic model for CP will help to unravel the complexities of this peculiar disease. Advanced genomic studies have helped us in many ways to understand the genetic basis of CP. For instance, genetic association studies have demonstrated that DNA variants (single nucleotide polymorphisms [SNPs]) play a crucial role in determining the susceptibility of an individual towards genetic alteration and their outcomes to an injury or external factors such as thrombosis or haemorrhage. These DNA variants influence the motor outcomes caused by the injury (haemorrhage or thrombosis). Single nucleotide polymorphisms produced through DNA variants are pivotal for the development of CP in a given individual. Copy number variants (CNVs) produced through duplication or deletion account for $20 \%$ of CP cases. CNVs studies have been found an interesting approach to delineate the complexities of the $\mathrm{CP}$ neurobiology by understanding the deleted/duplicated region of genomic interval. CNVs are crucial diagnostic/ prognostic tools for characterizing CP. In addition to CNVs, human-genome sequencing has enabled the 
identification of single gene mutations that can lead to the development of CP. These mutations are rare but have adverse outcomes [30].

\section{Genetic causes of cerebral palsy}

There are many stumbling blocks that have impeded genetic studies of CP. Insufficient sample size, heterogeneity of samples, and lack of functional validation are the major complications that have slowed the progress in this field. Therefore, only a handful of genes have been discovered so far; however, a number of studies are near to completion, which will broaden our understanding of CP. Gene association studies have been implemented to characterize single nucleotide variants that were found to increase the risk of developing CP. Meta-analysis studies have revealed that polymorphisms present in methylenetetrahydrofolate reductase, apolipoprotein $\mathrm{E}$ (ApoE), coagulation factor II-VII, interleukin 6 (IL-6), endothelial nitric oxide synthase, fibrinogen $\beta$-polypeptide, plasminogen activator inhibitor 1 , tumour necrosis factor $\alpha / \beta$, lymphotoxin $\alpha$ precursor, adductin-1, and $\beta$-adrenergic receptor were not involved in causing CP. However, IL-6 (rs1800795) was found to be significantly associated [47].

Apolipoprotein $\mathrm{E}$ (ApoE) plays a pivotal role in the central nervous system. APOE 22 and APOE 44 genotypes might serve as susceptibility factors in determining neurological outcomes after perinatal brain injury [22]. It has also been previously reported that children with APOE\&2/APOE\&4 alleles are more likely to die following cerebral injury in the uterus [42].

The presence of the APOE\&4 allele was strongly associated with more severe fine motor impairment among children with spastic unilateral cerebral palsy [26].

However, surprisingly, another team of researchers did not find any correlation and reported that APOE had no correlation in a direct way to the developmental sequelae of white or grey matter injuries in extremely preterm infants [5].

Osteopontin is a soluble immune factor involved in axonal regrowth and synaptogenesis after injury. An SNP, namely rs1126616 in the osteopontin gene, has been involved in nearly 700 Chinese patients [39]. These findings suggest that neuronal injuries can be a susceptible cause towards the development of $C P$, but further evidence is required to validate these results.
Courtesy of next-generation sequencing, a number of genes have been shown to contribute to the pathogenesis of CP. Emerging evidence is providing information about the underlying mechanisms of CP and how cellular pathways crosstalk for the regulation of oxidative stress and inflammation. Surprisingly, linking these genetic mutations to the vital neuronal pathways involved in CP can unravel their important role in neurodevelopmental processes.

Targeted next generation sequencing and whole exome sequencing have aided in the identification of point mutations in the genes ITPR 1, KCNC3, and SPTBN2 in subjects with ataxic CP [36].

Adaptor protein complex mutations have been linked with CP. AP-4 is a heterotetrameric adaptor protein complex produced by adaptor proteins, which modulates cell trafficking. AMPA (alpha-amino-3-hydroxy-5-methyl-4-isoxazolepropionic acid) receptor has been reported to be involved in receptor mediated glutamate excitotoxicity, which in turn promotes hypoxic-ischaemic white matter injury that in turn promotes CP. However AP-4 has been reported to control the cellular trafficking of AMPA and thus prevent excitotoxicity [32]. However, mutations in AP-4 complex can result in AMPA receptormediated glutamate excitotoxicity, which leads to hypoxic ischaemia. This mechanism has been shown in a number of CP cases. Patients harbouring AP-4 mutations are prone to develop quadriplegia, microcephaly, intellectual disability, and stunted growth. In addition to this, patients also develop lack of expressive speech [1]. Colpocephaly, decreased white matter, and cerebellar volume loss were also prominent [1].

\section{Preclinical studies}

Interestingly, studies have shown that perinatal cerebral hypoxic ischaemic injuries as well as inflammatory injuries are the main causes of cerebral palsy. PLPPR5 is an integral membrane molecule of the plasticity-related family of proteins [44]. It is expressed specifically in spinal cord as well as brain and drives the growth of the neurites. Moreover, it is significantly expressed in the brain, particularly in the regions of higher plasticity, such as the hippocampus. These signals are slightly low in the cerebellum, the cortex, as well as in the striata. Importantly, during the developmental process, expression of PLPPR5 is significant in the spinal cord. Evidently, 


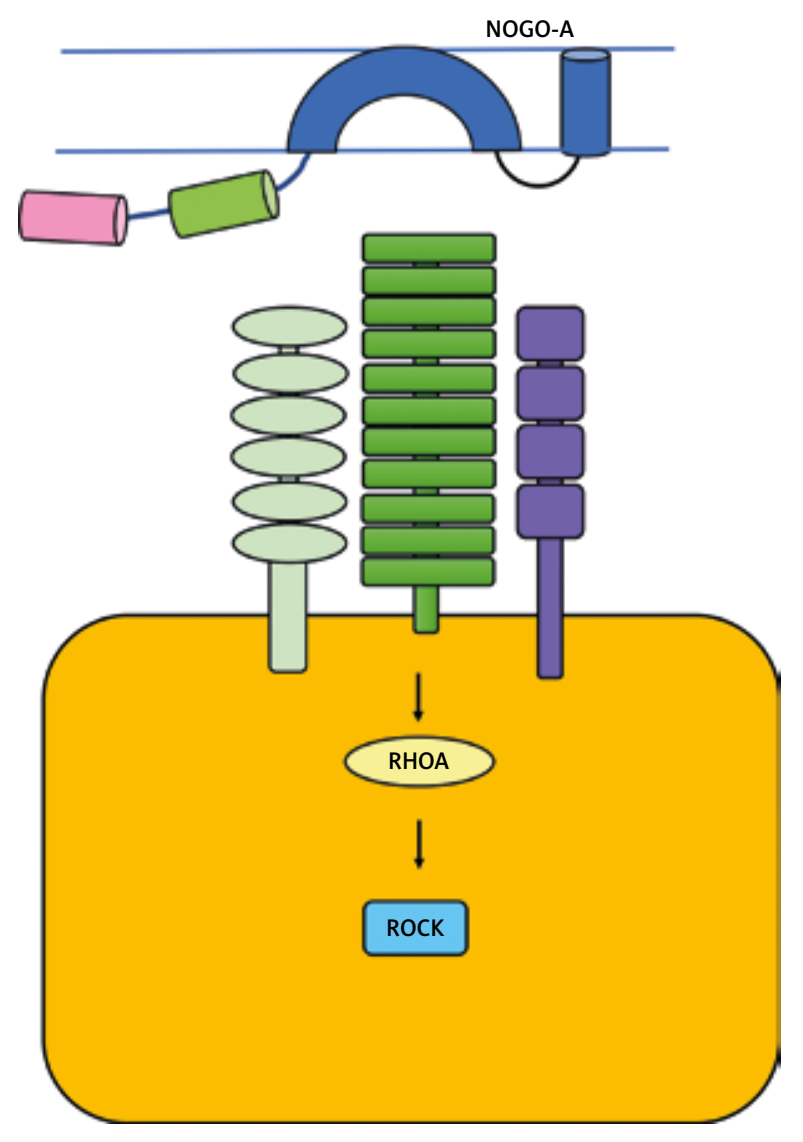

Fig. 1. NOGO-A on cell membranes interacts with multi-subunit receptors comprising ligand binding, signal transduction, and associated proteins.

expression of PLPPR5 mRNA was high in neuron-rich regions, particularly in medial motor nuclei. It has been suggested that PLPPR5 plays a critical part in the modulation of neurons. Experimental analysis indicates that wild-type mice having hypoxic-ischaemic brain injury show better performance as compared to PLPPR5-/- mice, irrespective of melatonin treatment. Moreover, melatonin treatment improves behaviour in the tests for wild-type animal models with hypoxic-ischaemic brain injuries, but not for PLPPR5-/- mice [44].

NOGO-A was characterized initially as a central nervous system-specific inhibitor of axonal regeneration. Scientists have sufficient evidence about regulatory roles of NOGO molecules and their specific receptors in precursor migration, growth of neurites, branching in the developing nervous system as well as a growth-inhibitory role during maturation of the

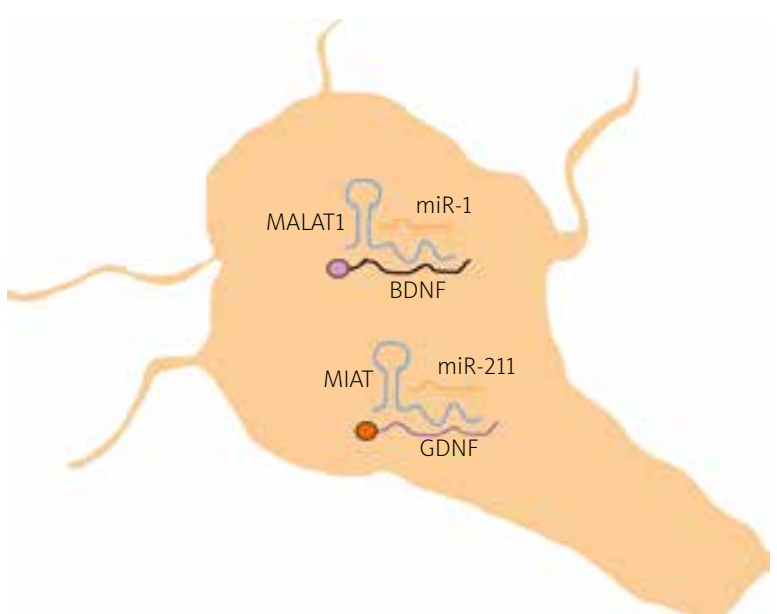

Fig. 2. MALAT1 and MIAT blocked neuronal cell death.

central nervous system. Constraint-induced movement therapy (CIMT) promoted the remodelling of neurons and functional reorganization through inhibition of NOGO-A/NgR/RhoA/ROCK signalling in hemiplegic cerebral palsy mice. CIMT promoted sprouting of the axons and post-stroke motor recovery by blockade of NOGO-A/RhoA/ROCK transduction cascade (Fig. 1) [28].

Non-coding RNAs have been reported to play a fundamental role in different diseases [2].

Vitamin $B_{1}$ and $B_{12}$ efficiently inhibited neuronal apoptosis by caucusing blockade of miRNA-mediated targeting of brain-derived neurotrophic factor (BDNF) [24]. miR-1 directly targeted BDNF and enhanced neuronal apoptosis, but MALAT1 interfered with miR-1-mediated targeting of BDNF (Fig. 2). MALAT1 and BDNF levels were found to be reduced in the rodent models of cerebral palsy, but they were restored by treatment with vitamin $B_{1}$ and $B_{12}$. More importantly, vitamin $B_{1}$ and $B_{12}$ did not enhance the levels of BDNF and MALAT1 in mice treated with short interfering RNAs for MALAT1 [24]. Moreover, circumstantial evidence indicates that MIAT overexpression reduces apoptotic death of Neuro2A cells [23]. Glial cell line-derived neurotrophic factor (GDNF) was essential for the protection of neurons. MIAT inhibited miR-211-mediated targeting of GDNF (Fig. 2). MIAT overexpression improved motor function of rats with hypoxic-ischaemic $(\mathrm{HI})$ injuries. MIAT was transfected into mice. Long non-coding RNA MIAT was upregulated in striatal tissues of lentiMIAT-treated groups, while miR-211 was reported 
to be reduced considerably. Importantly, expression of GDNF was upregulated in striatal tissues of lentiMIAT-treated groups. MIAT antagonized miR-211-mediated targeting of GDNF [23].

Natural products can be considered s key to different questions. Natural products have been shown to be highly effective against a broad spectrum of diseases $[6,15,27,38]$.

Gastrodin is a traditional Chinese medicine [18]. Gastrodin is a biologically active product and promotes gross and fine motor performance. Gastrodin prompts the differentiation of macrophage into $M 2$ phenotype by upregulation of BCL6. Gastrodin evidently suppresses $\mathrm{H}_{2} \mathrm{O}_{2}$-induced apoptotic death in RAW264.7 cells, and these effects are abrogated in BCL6-silenced cells. Subcutaneous injections of Gastrodin at acupuncture points were reported to be an effective, cost-effective, and less-invasive method for the improvement of motor performance in cerebral palsy patients [18].

Intracerebrally transplanted $\mathrm{CD}_{34}{ }^{+}$haematopoietic stem cells effectively improved sensorimotor performances in hypoxia-ischaemic injury-induced mice. Notably, the treatment also considerably improved hypoxia-ischaemic injury-induced exploratory behaviours and locomotion impairments [7].

Mouse pups were subjected to hypoxia-ischaemia and lipopolysaccharide-induced inflammation (HIL) in an experimental model of cerebral palsy, leading to neuronal injuries in periventricular white matter, neocortex, and hippocampus [41]. Rapamycin (sirolimus), a macrolide compound, has been shown to be an effective inhibitor of mTOR. Rapamycin remarkably blocked laminar disorganization following HIL in the hippocampus of experimental animals. The structure of the hippocampus 1 week and 1 month after treatment with rapamycin was intact in HIL-induced models of CP [41].

Nanotechnological delivery of therapeutic agents has gained interest $[25,34,45,46]$.

CD11b is expressed on the surface of activated microglia that are involved in neurodegeneration and inflammation [21]. It is upregulated by oxidative stress and plays a crucial role in the exacerbation of the neuro-inflammatory processes. Dendrimer-based $\mathrm{N}$-acetyl-l-cysteine (NAC) treatment suppressed neuro-inflammation and markedly improved motor functions in the CP kits. Neuroinflammation is associated with loss of myelination, resulting in the characteristic white matter injuries observed in CP.
Dendrimer-based NAC led to a significant increase in myelin in the kits [21].

A wealth of information has shown that activation of microglia can induce neuronal damage through the release of proinflammatory cytokines, ROS, and free radicals. Dendrimer-minocycline conjugates facilitated minocycline to cross the bloodbrain barrier and to target activated microglia at the injury sites [40].

\section{Conclusions}

Accumulating evidence has begun to shed light on the involvement of genetics in CP. Microarray analysis of CP patients with unknown aetiology can be used as a unifying diagnostic tool for the detection CP. Moreover, progress in whole exome sequencing could be implemented to further delineate the underlying genetic predispositions responsible for CP. The rapidly expanding list of regulators of CP will enable scientists to unravel the complexities of CP and devise new diagnostic strategies that will enable rapid detection. But still many validating studies are needed. Collectively, a large number of genomic discoveries followed by extensive in vitro and in vivo studies will broaden our understanding of the molecular mechanism involved in CP. This will help in the treatment of fundamental pathophysiology of CP at an early stage.

\section{Disclosure}

The authors report no conflict of interest.

\section{References}

1. Abou Jamra R, Philippe O, Raas-Rothschild A, Eck SH, Graf E, Buchert R, Borck G, Ekici A, Brockschmidt FF, Nöthen MM. Adaptor protein complex 4 deficiency causes severe autosomal-recessive intellectual disability, progressive spastic paraplegia, shy character, and short stature. Am J Hum Genet 2011; 88: 788-795.

2. Adylova A, Mukhanbetzhanovna AA, Attar R, Yulaevna IM, Farooqi AA. Regulation of TGF/SMAD signaling by long non-coding RNAs in different cancers: Dark Knight in the Castle of molecular oncology. Noncoding RNA Res 2021; 6: 23-28.

3. Badawi N, Keogh JM. Causal pathways in cerebral palsy. J Paediatr Child Health 2013; 49: 5-8.

4. Baier RJ. Genetics of perinatal brain injury in the preterm infant. Front Biosci 2006; 11: 1371-1387.

5. Blackman JA, Gordish-Dressman H, Bao Y, Matsumoto JA, Sinkin RA. The apolipoprotein gene and recovery from brain injury among extremely preterm infants. Neonatology 2014; 105: 227-229. 
6. Butt G, Faroogi AA, Adylova A, Attar R, Yilmaz S, Konysbayevna KK, Sabitaliyevich UY, Gasparri ML, Xu B. Vitamin C as an anticancer agent: regulation of signaling pathways. Curr Top Med Chem 2020; 20: 1868-1875.

7. Chang Y, Lin S, Li Y, Liu S, Ma T, Wei W. Umbilical cord blood CD34+ cells administration improved neurobehavioral status and alleviated brain injury in a mouse model of cerebral palsy. Childs Nerv Syst 2021; 37: 2197-2205.

8. Corbett MA, van Eyk CL, Webber DL, Bent SJ, Newman M, Harper K, Berry JG, Azmanov DN, Woodward KJ, Gardner AE. Pathogenic copy number variants that affect gene expression contribute to genomic burden in cerebral palsy. NPJ Genom Med 2018; 3: 1-9.

9. Ellenberg JH, Nelson KB. The association of cerebral palsy with birth asphyxia: a definitional quagmire. Dev Med Child Neuro 2013; 55: 210-216.

10. Emrick LT, DiCarlo SM. The expanding role of genetics in cerebral palsy. Phys Med Rehabil Clin N Am 2020; 31: 15-24.

11. Faroogi AA, Attar R, Yulaevna IM, Berardi R. Interaction of long non-coding RNAs and circular RNAs with microRNAs for the regulation of immunological responses in human cancers. Semin Cell Dev Biol 2021; S1084-9521(21)00138-5.

12. Faroogi AA, Fayyaz S, Poltronieri P, Calin G, Mallardo M. Epigenetic deregulation in cancer: Enzyme players and non-coding RNAs. Semin Cancer Biol 2020; S1044-579X(20)30170-X.

13. Faroogi AA, Li Y, Sarkar FH. The biological complexity of RKIP signaling in human cancers. Exp Mol Med 2015; 47: e185.

14. Farooqi AA, Naureen H, Attar R. Regulation of cell signaling pathways by circular RNAs and microRNAs in different cancers: Spotlight on Wnt/ $\beta$-catenin, JAK/STAT, TGF/SMAD, SHH/ GLI, NOTCH and Hippo pathways. Semin Cell Dev Biol 2021; 13 S1084-9521(21)00075-6.

15. Farooqi AA, Naureen H, Zahid R, Youssef L, Attar R, Xu B. Cancer chemopreventive role of fisetin: Regulation of cell signaling pathways in different cancers. Pharmacol Res 2021; 172 105784.

16. Farooqi AA, Nayyab S, Martinelli C, Berardi R, Katifelis H, Gazouli M, Cho WC. Regulation of hippo, TGF $\beta / S M A D$, Wnt/ $\beta$-catenin, JAK/STAT, and NOTCH by long non-coding RNAs in pancreatic cancer. Front Oncol 2021; 11: 657965.

17. Farooqi AA, Qureshi MZ, Coskunpinar E, Naqvi SK, Yaylim I, Ismail M. MiR-421, miR-155 and miR-650: emerging trends of regulation of cancer and apoptosis. Asian Pac J Cancer Prev 2014; 15: 1909-1912.

18. Jia J, Shi X, Jing X, Li J, Gao J, Liu M, Lin Cl, Guo X, Hua Q. BCL6 mediates the effects of Gastrodin on promoting M2-like macrophage polarization and protecting against oxidative stress-induced apoptosis and cell death in macrophages. Biochem Biophys Res Commun 2017; 486: 458-464.

19. Jiao Z, Jiang Z, Wang J, Xu H, Zhang Q, Liu S, Du N, Zhang Y, Qiu H. Whole-genome scale identification of methylation markers specific for cerebral palsy in monozygotic discordant twins. Mo Med Rep 2017; 16: 9423-9430.

20. Jin SC, Lewis SA, Bakhtiari S, Zeng X, Sierant MC, Shetty S, Nordlie SM, Elie A, Corbett MA, Norton BY, van Eyk CL, Haider S, Guida BS, Magee H, Liu J, Pastore S, Vincent JB, Brunstrom-Hernandez J, Papavasileiou A, Fahey MS, Berry JG, Harper K, Zhou C,
Zhang J, Li B, Zhao H, Heim J, Webber DL, Frank MSB, Xia L, Xu Y, Zhu D, Zhang B, Sheth AH, Knight JR, Castaldi C, Tikhonova IR, López-Giráldez F, Keren B, Whalen S, Buratti J, Doummar D, Cho M, Retterer K, Millan F, Wang Y, Waugh JL, Rodan L, Cohen JS, Fatemi A, Lin AE, Phillips JP, Feyma T, MacLennan SC, Vaughan S, Crompton KE, Reid SM, Reddihough DS, Shang Q, Gao C, Novak I, Badawi N, Wilson YA, Mclntyre SJ, Mane SM, Wang X, Amor DJ, Zarnescu DC, Lu Q, Xing Q, Zhu C, Bilguvar K, Padilla-Lopez S, Lifton RP, Gecz J, MacLennan AH, Kruer MC. Mutations disrupting neuritogenesis genes confer risk for cerebral palsy. Nat Genet 2020; 52: 1046-1056.

21. Kannan S, Dai H, Navath RS, Balakrishnan B, Jyoti A, Janisse J, Romero R, Kannan RM. Dendrimer-based postnatal therapy for neuroinflammation and cerebral palsy in a rabbit model. Sci Transl Med 2012; 4: 130ra46.

22. Kuroda MM, Weck ME, Sarwark JF, Hamidullah A, Wainwright MS. Association of apolipoprotein E genotype and cerebral palsy in children. Pediatrics 2007; 119: 306-313.

23. Li EY, Zhao PJ, Jian J, Yin BQ, Sun ZY, Xu CX, Tang YC, Wu H. LncRNA MIAT overexpression reduced neuron apoptosis in a neonatal rat model of hypoxic-ischemic injury through miR211/GDNF. Cell Cycle 2019; 18: 156-166.

24. Li EY, Zhao PJ, Jian J, Yin BQ, Sun ZY, Xu CX, Tang YC, Wu H. Vitamin B1 and B12 mitigates neuron apoptosis in cerebral palsy by augmenting BDNF expression through MALAT1/miR-1 axis. Cell Cycle 2019; 18: 2849-2859.

25. Li X, Xu H, Li C, Qiao G, Farooqi AA, Gedanken A, Liu X, Lin X. Zinc-doped copper oxide nanocomposites inhibit the growth of pancreatic cancer by inducing autophagy through AMPK/mTOR pathway. Front Pharmacol 2019; 10: 319

26. Lien E, Andersen GL, Bao Y, Gordish-Dressman H, Skranes JS, Vik T, Blackman JA. Apolipoprotein E polymorphisms and severity of cerebral palsy: a cross-sectional study in 255 children in Norway. Dev Med Child Neurol 2013; 55: 372-377.

27. Lin X, Faroogi AA. Cucurbitacin mediated regulation of deregulated oncogenic signaling cascades and non-coding RNAs in different cancers: Spotlight on JAK/STAT, Wnt/ $\beta$-catenin, mTOR, TRAIL-mediated pathways. Semin Cancer Biol 2021; 73: 302309

28. Liu LR, Wang YX, He L, Xu YX, Huang JY, Peng TT, Yang XB, Pan J, Tang HM, Xu KS. Constraint-induced movement therapy promotes neural remodeling and functional reorganization by overcoming Nogo-A/NgR/RhoA/ROCK signals in hemiplegic cerebral palsy mice. Neurorehabil Neural Repair 2021; 35: 145 157.

29. Liu PF, Farooqi AA, Peng SY, Yu TJ, Dahms HU, Lee CH, Tang JY, Wang SC, Shu CW, Chang HW. Regulatory effects of noncoding RNAs on the interplay of oxidative stress and autophagy in cancer malignancy and therapy. Semin Cancer Biol 2020; 27: S1044-579X(20)30214-5.

30. McMichael G, Girirajan S, Moreno-De-Luca A, Gecz J, Shard C, Nguyen LS, Nicholl J, Gibson C, Haan E, Eichler E. Rare copy number variation in cerebral palsy. Eur J Hum Genet 2014; 22: 40-45.

31. Mohandas N, Bass-Stringer S, Maksimovic J, Crompton K, Loke YJ, Walstab J, Reid SM, Amor DJ, Reddihough D, Craig JM. Epigenome-wide analysis in newborn blood spots from mono- 
zygotic twins discordant for cerebral palsy reveals consistent regional differences in DNA methylation. Clin Epigenetics 2018; 10: 25 .

32. Moreno-De-Luca A, Helmers SL, Mao H, Burns TG, Melton AM, Schmidt KR, Fernhoff PM, Ledbetter DH, Martin CL. Adaptor protein complex-4 (AP-4) deficiency causes a novel autosomal recessive cerebral palsy syndrome with microcephaly and intellectual disability. J Med Genet 2011; 48: 141-144.

33. Moreno-De-Luca A, Millan F, Pesacreta DR, Elloumi HZ, Oetjens MT, Teigen C, Wain KE, Scuffins J, Myers SM, Torene RI. Molecular diagnostic yield of exome sequencing in patients with cerebral palsy. JAMA 2021; 325: 467-475.

34. Nogueira DR, Scheeren LE, Macedo LB, Marcolino Al, Pilar Vinardell M, Mitjans M, Rosa Infante M, Farooqi AA, Rolim CM. Inclusion of a $\mathrm{pH}$-responsive amino acid-based amphiphile in methotrexate-loaded chitosan nanoparticles as a delivery strategy in cancer therapy. Amino Acids 2016; 48: 157-168.

35. Paneth N, Hong T, Korzeniewski S. The descriptive epidemiology of cerebral palsy. Clin Perinatol 2006; 33: 251-267.

36. Parolin Schnekenberg R, Perkins EM, Miller JW, Davies WI, D’Adamo MC, Pessia M, Fawcett KA, Sims D, Gillard E, Hudspith K. De novo point mutations in patients diagnosed with ataxic cerebral palsy. Brain 2015; 138: 1817-1832.

37. Poltronieri P, Mezzolla V, Farooqi AA, Di Girolamo M. NAD precursors, mitochondria targeting compounds and ADPribosylation inhibitors in treatment of inflammatory diseases and cancer. Curr Med Chem 2021; Jan 18.

38. Shahwar D, Iqbal MJ, Nisa MU, Todorovska M, Attar R, Sabitaliyevich UY, Farooqi AA, Ahmad A, Xu B. Natural product mediated regulation of death receptors and intracellular machinery: fresh from the pipeline about TRAIL-mediated signaling and natural TRAIL sensitizers. Int J Mol Sci 2019; 20: 2010.

39. Shang Q, Zhou C, Liu D, Li W, Chen M, Xu Y, Wang F, Bi D, Zhang X, Zhao X. Association between osteopontin gene polymorphisms and cerebral palsy in a Chinese population. Neuromolecular Med 2016; 18: 232-238.

40. Sharma R, Kim SY, Sharma A, Zhang Z, Kambhampati SP, Kannan S, Kannan RM. Activated microglia targeting dendrimer-minocycline conjugate as therapeutics for neuroinflammation. Bioconjug Chem 2017; 28: 2874-2886.

41. Srivastava IN, Shperdheja J, Baybis M, Ferguson T, Crino PB. mTOR pathway inhibition prevents neuroinflammation and neuronal death in a mouse model of cerebral palsy. Neurobiol Dis 2016; 85: 144-154.

42. Stoknes M, Lien E, Andersen GL, Bao Y, Blackman JA, Lie RT, Vik T. Child apolipoprotein E gene variants and risk of cerebral palsy: estimation from case-parent triads. Eur J Paediatr Neurol 2015; 19: 286-291.

43. Strijbis EM, Oudman I, van Essen P, MacLennan AH. Cerebra palsy and the application of the international criteria for acute intrapartum hypoxia. Obstet Gynecol 2006; 107: 1357-1365.

44. Sun Y, Ma L, Jin M, Zheng Y, Wang D, Ni H. Effects of melatonin on neurobehavior and cognition in a cerebral palsy model of plppr5-/- mice. Front Endocrinol (Lausanne) 2021; 12: 598788.

45. Wen R, Umeano AC, Chen P, Farooqi AA. Polymer-based drug delivery systems for cancer. Crit Rev Ther Drug Carrier Syst 2018; 35: 521-553.
46. Wen R, Umeano AC, Kou Y, Xu J, Farooqi AA. Nanoparticle systems for cancer vaccine. Nanomedicine (Lond) 2019; 14: 627648.

47. Wu D, Zou YF, Xu XY, Feng XL, Yang L, Zhang GC, Bu XS, Tang JL. The association of genetic polymorphisms with cerebral palsy: a meta-analysis. Dev Med Child Neurol 2011; 53: 217-225.

48. Zhang Z, Lin YA, Kim SY, Su L, Liu J, Kannan RM, Kannan S. Systemic dendrimer-drug nanomedicines for long-term treatment of mild-moderate cerebral palsy in a rabbit model. J Neuroinflammation 2020; 17: 319 